\title{
Design, Fabrication and Evaluation of a Home-made Floor Polisher
}

\author{
Mark Paul A. Covita ${ }^{1, *}$ and Reizel Emilfroi R. Villanueva ${ }^{2}$ \\ ${ }^{1}$ Faculty of Industrial Technology, Cagayan State University, Cagayan State University, Sanchez Mira, Cagayan, \\ Philippines; markpaulcovita012414@gmail.com; \\ ²Cadet at Hartmann Crew Philippines; emilfroireizel@gmail.com
}

\begin{abstract}
Objectives: This research study determined the feasibility of a home-made floor polisher. Specifically, it designed, fabricated and evaluated the home-made floor polisher and determined the machine efficiency and its cost. Methods and analysis: One may find it more effective to use an electric floor polisher to clean and polish rather than brushing and polishing manually using the traditional coconut husk floor scrub. The device was tested and compared with the commercial one. They were tested in the same area and dirtiness. The data gathered comprised of time consumed in polishing and concluded that improvised floor polisher is effective in polishing and is cost-effective. Findings: Based on the data gathered, the home-made floor polisher is more efficient than the commercial polisher in terms of cleaning efficiency and capability. It also helps reduce waste because the parts are made of recycled items from the defective washing machine or any devices used in electric motors. It can also help to lessen the pollution as the husk can have myriad uses instead of burning. The home-made floor polisher can help people in our community for a faster completion of assigned task in scrubbing the floor with less energy input. Instead of buying the materials needed, the motor from a defective washing machine appropriate for the use of floor polisher is a good way for minimising expenses. The developed home-made floor polisher will serve as a basis not only for students, teachers or instructors but also future researchers to be able to build, develop and improvise more products relative to the materials used in the study. Application: The improvised home-made floor polisher is very efficient in terms of its power consumption, cleaning efficiency and capability. It is very effective in polishing floor cement compared to commercial one.
\end{abstract}

Keywords: Fabrication, Evaluation, Efficiency, Polisher, Brushing, Development.

\section{Introduction}

The coconut tree is very abundant in our community. It is popularly known for its many useful parts from its coo lumber to its fruit. Many products can be produced from the mature to the old coconut, but this old or brown colored coconut fruit has still been used. It can be used as a floor scrub. 1 Coconut husk floor scrub has been used for years by Filipinos in polishing wood and cement flooring. By just cutting the old coconut into halves, it can be directly used for polishing and brushing dust. Coconut is known for its great versatility as seen in the many uses of its different parts. The coconut husk, also known as coir, has become a very useful substance in the light of today's environmental and economic concerns. This may be surprising, but coir is a key component in the production of some popular products. Carpets, rugs, doormats, mattresses, car seat covers, brushes, bristles and flower pots are just some items that can be made from coconut fibers. $\underline{2}$

It has been reported that the husk can be used as a floor scrubber. Locally known as "bunot", it is a traditional tool for polishing wooden floors. You can even mirror the image with the shiny floor with the help of "bunot" or coconut husk. Filipinos use coconut husk to scrub the wooden floor. It is a form of a daily routine or exercise prior to taking a bath early in the morning. A long time before the floor polisher devices were invented, people in

${ }^{*}$ Author for correspondence 
the Philippines used the cheapest way of making the floor shiny through "bunot" or coconut husk. For those people who cannot afford the modern polisher, they still have the natural alternative. $\underline{3}$

From the olden times to the modernised world, people have been innovating and improvising machines and other materials to cope up with the need of our community. Our traditional floor polisher (coconut husk floor scrub) has been altered or influenced by foreign countries that later introduced the electric floor polisher. When cleaning at home, using the traditional coconut husk floor scrub consumes much time, especially when cleaning alone. To ensure proper floor maintenance, cleaning professionals must be clear which areas need the most frequent attention. This ensures the floors are properly maintained and can help reduce the frequency of floor restoration. $\underline{4}$

However, floor polisher nowadays is very expensive. A brand-new Wilson floor polisher model 406-16in is worth 32,249.75 pesos (Ace Hardware). That is heavy on the budget.

This study was conducted to improvise a floor polisher using recycled items. The coconut husk used as floor polisher scrub can be replaced or can easily be substituted by a mapping cloth.

\section{Materials and Method}

This section presents the researcher's methods and procedures that were utilised in the study. It includes the materials needed, general procedure and assembly procedure.

\subsection{Materials of the Study}

Table 1 shows the materials that were secured from a local store and some parts were fabricated. The home-made floor polisher consisted of the following parts and their corresponding functions.

\subsection{General Procedure}

The motor used in the fabrication of the home-made floor polisher was gathered from a junk shop along with its capacitor and reused motor from a defective washing machine. The motor was tested to see if it actually works. The wiring connections and defective parts of the motor were fixed together with the capacitor connection.
Table 1. Materials needed

\begin{tabular}{|l|l|}
\hline Bought materials & Fabricated and re-used \\
\hline Electric wash motor & Motor frame \\
\hline Capacitor & Coconut husk \\
\hline Flat wire & $\begin{array}{l}\text { Coconut husk floor scrub } \\
\text { brush }\end{array}$ \\
\hline Male plug & Reused speaker stand \\
\hline Wheels & Reused floor polisher handle \\
\hline $\begin{array}{l}\text { Bolt and nut (\#10 - 1 inch, } \\
3 \text { inch) }\end{array}$ & \\
\hline $\begin{array}{l}\text { Galvanised iron (\# } 14 \text { GI } \\
\text { sheet) }\end{array}$ & \\
\hline Welding rod (ordinary) & \\
\hline $\begin{array}{l}\text { Spray paint (maroon no. 38, } \\
\text { gray, clear) }\end{array}$ & \\
\hline
\end{tabular}

\subsection{Preparing the Coconut Husk Floor Scrub Brush}

Figure 1 shows the coconut scrub being substituted for the brush. A matured coconut was cut horizontally into halves, then the coconut shell was removed. The husk was cut again into the desired size. The coconut husk was mounted to the floor polisher. The tip of the coconut husk was pounded so that it was easier to form a brush and would have less friction when in contact with the floor.

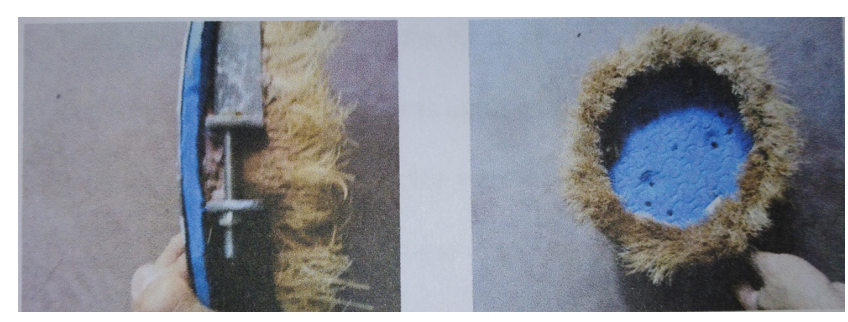

Figure 1. Coconut substituted as the brush.

\subsection{Making the Circular Clamp for the}

\section{Floor Polisher}

Figure 2 shows the construction of the motor attachment. Using a metal scissor, metal sheets were cut to form a floor scrub. The old propeller of the motor was reused so it had a perfect fit for the motor. The propeller which originally had some fins was grinding for it to have a flat and smooth surface. A rivet was used to attach the circular black sheet to the reused propeller motor.

Figure 3 shows the clamp of the coconut husk composed of two flat bars; each flat bar was shaped circularly. One 




Figure 2. Making the circular clamp for the floor polisher.

bar had a smaller circumference than the other so that the coconut husk could be clamped together. The inner flat bar with a smaller circumference was welded to its tip so it cannot move. With the use of bolt and nut, it was screwed to the black sheet. The outer flat bar was not welded to its tip; instead a bolt and nut were used to lock it. In its tip, a small portion was bend outward. Two identical holes were drilled between the flat bars in its half portion and a bolt and nut were used to lock it.
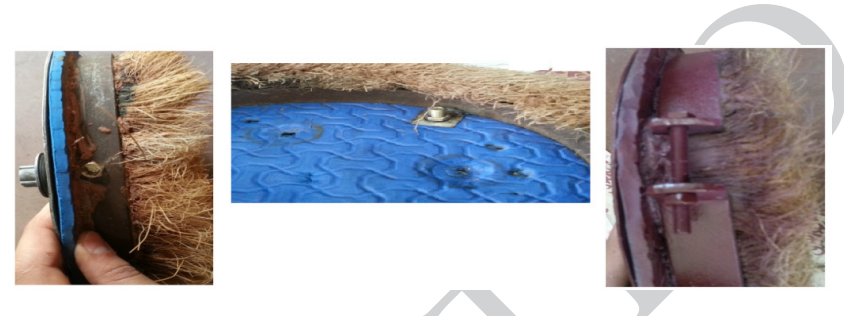

Figure 3. Clamp of the coconut husk composed of two flat bars.

\subsection{Construction Frame of the Motor}

Figure 4 shows the frame constructed following the shape of the motor using a light steel frame. The wirings used in the motor were also measured, then it was welded and screwed using the bolt and nut (bolt head \#10) to secure the attachments.
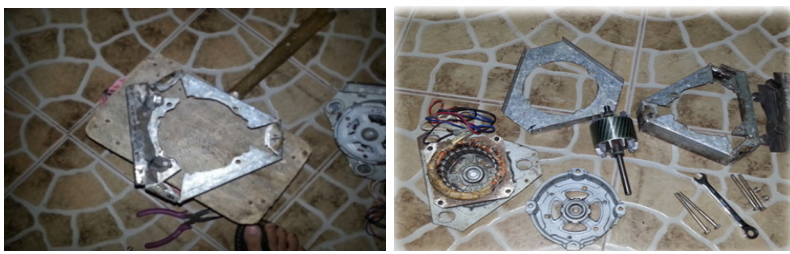

Figure 4. Frame constructed following the shape of the motor using a light steel frame.

\subsection{Mounting the Motor to Its Frame}

Figure 5 shows the electric motor mounted and screwed using bolt and nut to its frame.

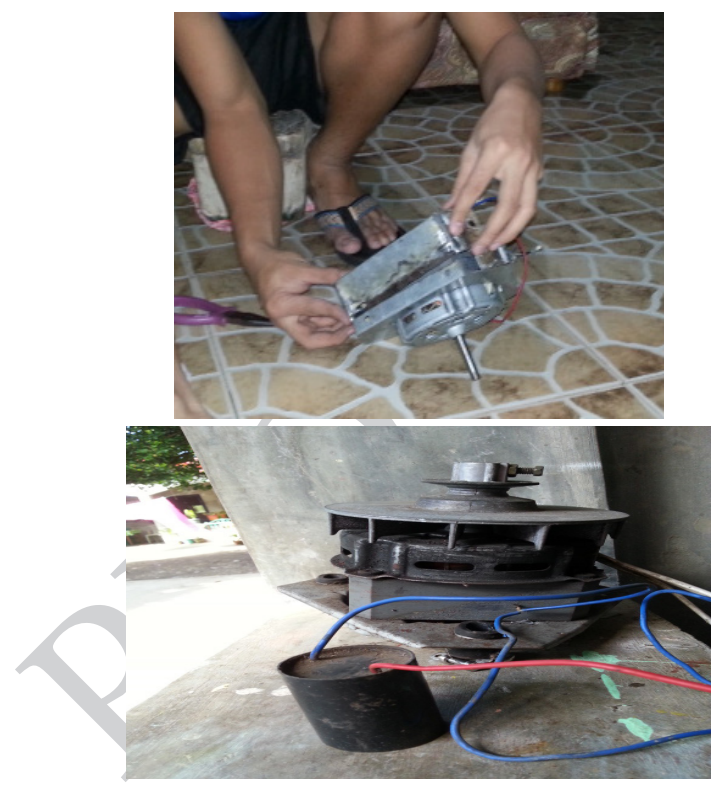

Figure 5. Electric motor mounted and screwed using bolt and nut to its frame.

\subsection{Attachment of the Inverted $\mathrm{C}$ to the}

\section{Handle as Wheel of the Motor Frame}

Figure 6 shows a wheel mounted in a motor frame; a " $\mathrm{C}$ shape" flat bar was constructed. This served as attachment between the electric motor frame and the motor to link the handle and the wheels. For the handle to be attached, two flat bars were welded at the center with a distance of two inches apart and drilled at the center for the handle to tighten.

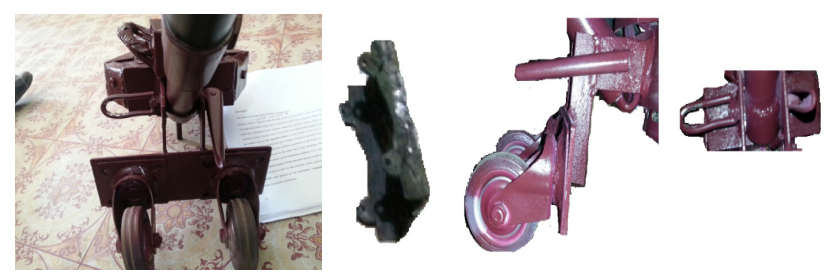

Figure 6. Wheel mounted in a motor frame.

To attach the wheel, two flat bars were welded downward to the tip of the handle flat bar attachment. The two wheels had a width of 10 inches and a height of 2.5 inches. The height of the wheels was equal to the coconut husk brush head. These wheels function to assist 
the weight of the motor itself in order for the motor to spin the brush and decrease friction between the coco husk and the floor and to easily navigate the floor polisher.

\subsection{Wiring Connections}

Figure 7 shows the wiring connection of the electric motor composed of a capacitor that was connected to the motor which made the motor spin clockwise or counterclockwise. A push button switch mechanism was used for the operation of the clockwise and the counterclockwise rotation. When the push button was released, the motor automatically turned on; a wire connecting the motor and the male plug was used so it can be inserted into a receptacle or socket.


Figure 7. Wiring connection of the electric motor composed of a capacitor connected to the motor.

\subsection{Floor Polisher's Finishing}

After the coconut brush was fixed to its circular clamp, the brush was pasted using all-purpose epoxy. The clamp was mounted to the motor. An adjustable lever was screwed. It was connected to the motor frame so that the handle could be adjusted and moved forward and backward. A reused speaker stand was used as the body of the handle, connected diagonally to that of the metal frame. A reused floor polisher handle was used as the handle. It was attached on top of the speaker stand to act as the steering wheel for the floor polisher so that it can be controlled. The switch was fastened on the left and right side of the handle.

\subsection{Steps in Assembling}

1. The frame was mounted to the motor. A bolt and a nut were used to secure it with bolt head $\# 10$.

2. The reused speaker stand was attached to the $C$ flat bar, mounted to the motor frame and where the wheels were attached.

3. The blue wire of the capacitor and the motor were attached to a wire that connects the male plug. The black wire of the motor was attached to the other wire of the male plug. The wire was inserted inside the speaker stand. A capacitor box was built to protect the capacitor. It was placed on top of the motor.

4. The brush was inserted to the motor and was tightened through a bolt and a nut as shown in Figure 8.

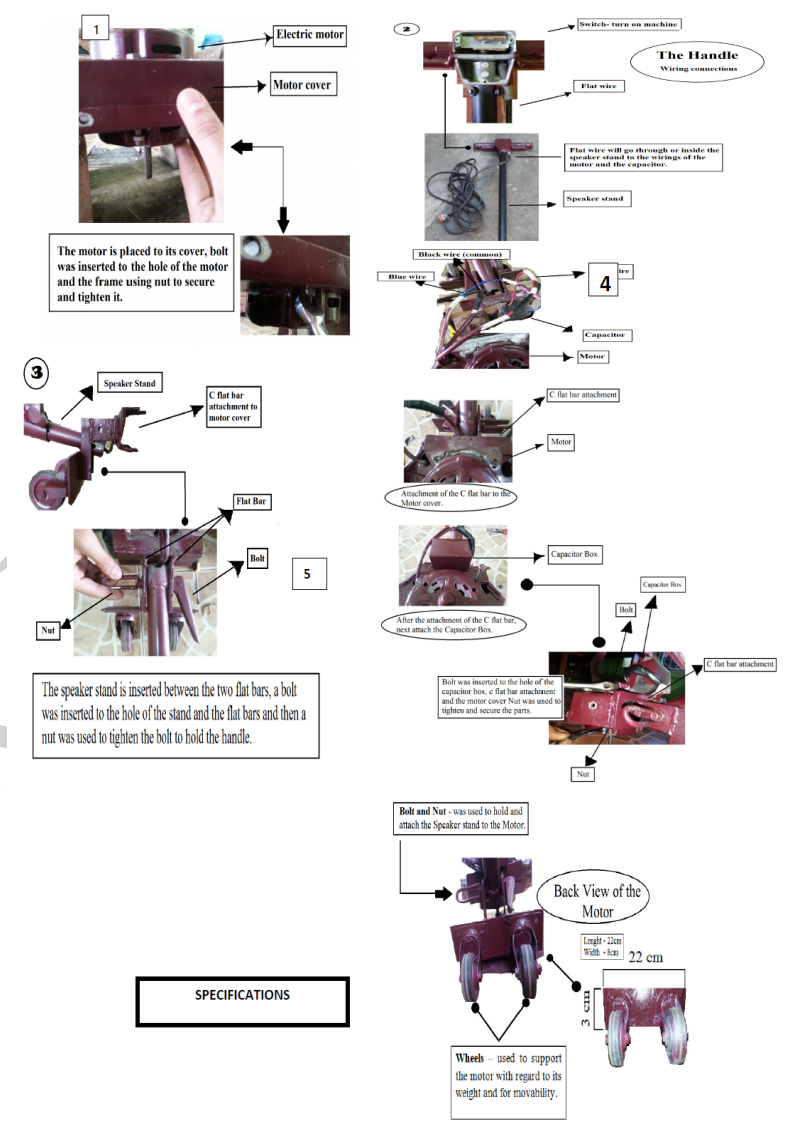

Figure 8. Assembly of the home-made floor polisher.

\subsection{Safety Precaution}

When using this electrical appliance, simple security precautions should be observed, including the following:

1. Read all instructions before using the gadget.

2. Do not submerge the wire and the male plug in water or in any other liquid to avoid electric shock.

3. Check the cord for open wiring. Then bind it with an adhesive tape.

4. Unplug the appliance when not in use.

5. Close supervision is necessary when this appliance is used by or near children.

6. Avoid contact with moving parts especially the brush head. 
7. Do not operate this appliance with a damaged cord or plug.

8. Do not touch the surface of the device when in use.

\subsection{Data Gathering Procedure}

For the evaluation of the performance of the home-made floor polisher, the machine was operated to polish cement flooring.

\subsection{Testing and Operation}

The floor polisher was tested in terms of the amount of time consumed in polishing the floor.

\subsection{Performance Evaluation}

Machine efficiency was determined by the cleanliness of the scrubbed and polished flooring and by its speed in polishing.

\subsection{Preliminary Test}

Before the conduct of the final test, an initial test was conducted to determine the defective parts of the floor polisher.

\subsection{Final Test}

The final test was conducted by testing the time needed to scrub and polish cement flooring.

\subsection{Statistical Analysis}

Data was gathered during the final testing and analyzed using descriptive, inferential statistics.

\section{Results and Discussions}

This section presents the analysis and interpretation of data concerning the study, specification of materials, electric wiring diagram and the final design.

Table 2 shows that the commercial floor polisher consumed more time than the home-made floor polisher. This depends however on the user's capability to operate the machine.

The commercial floor polisher consumed 9.5 minutes in polishing, while the home-made floor polisher consumed 8.6 minutes in polishing. It is further presented that the improvised floor polisher is efficient in terms of its cleaning capability because the floor polished by the
Table 2. Efficiency of the home-made floor polisher

\begin{tabular}{|l|l|l|}
\hline Type of machine & $\begin{array}{l}\text { Time consumed } \\
(\mathbf{m i n})\end{array}$ & $\begin{array}{l}\text { Area of the } \\
\text { floor }(\mathbf{m})\end{array}$ \\
\hline $\begin{array}{l}\text { Home-made floor } \\
\text { polisher }\end{array}$ & 8.6 & $8.35 \times 2.74$ \\
\hline $\begin{array}{l}\text { Commercial floor } \\
\text { polisher }\end{array}$ & 9.5 & $8.35 \times 2.74$ \\
\hline
\end{tabular}

home-made machine is shinier and cleaner as compared to the commercial floor polisher, as shown in Figure 9.


Input 220 volt

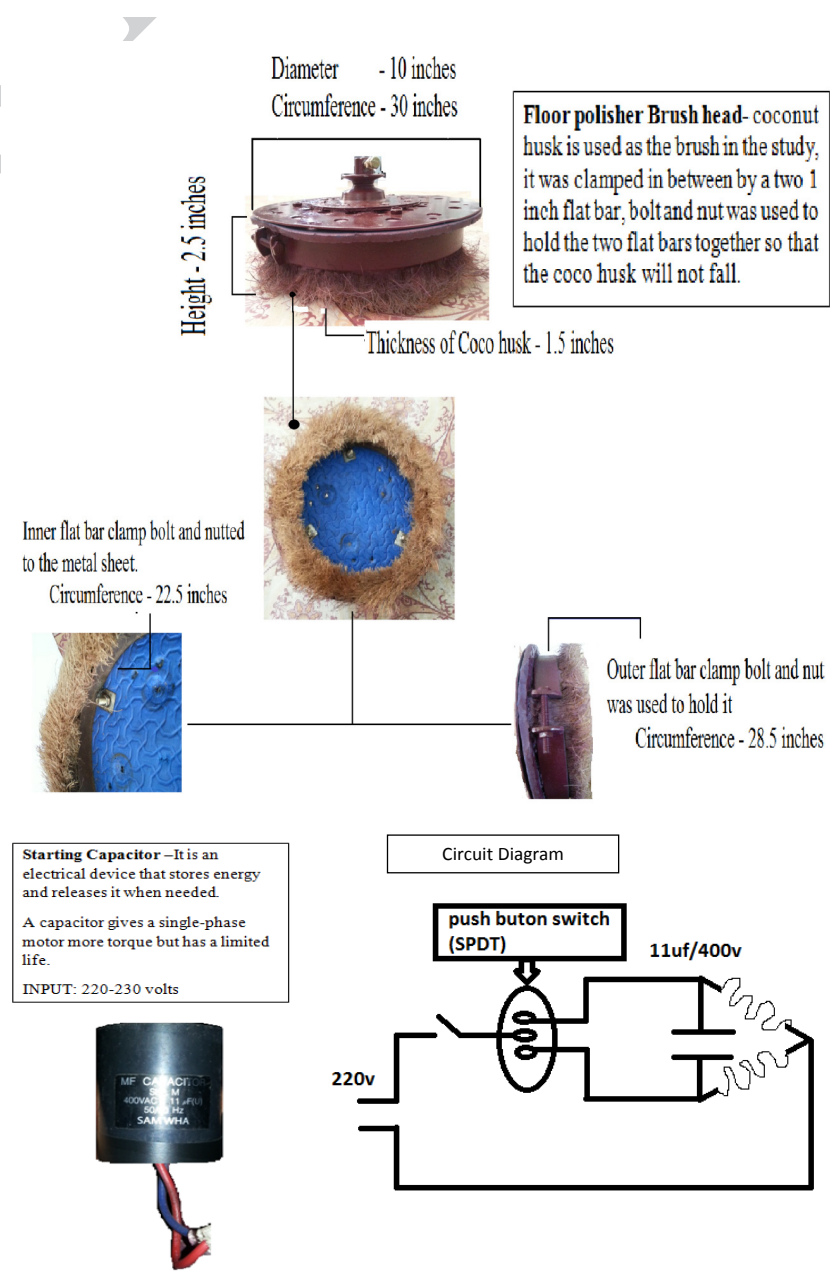





Figure 9. Components of the home-made floor polisher.

\section{Conclusion}

Based on the results and findings, the home-made floor polisher is more efficient than the commercial prototype in terms of its cleaning capability. It is also cost-effective. Compared to the commercial prototype, the parts are easy to replace.

\section{Recommendation}

1. The researcher recommends the use of a stronger motor to polish more efficiently. It is also recommended to use a light-weight motor cover for the motor.

2. A protective covering should be constructed to cover the polisher brush.

3. The rotation of the brush should be made clockwise and counter-clockwise if wash motor was used to help maneuver the machine in any direction.

4. A new design that is self-maneuvering should be fabricated, one that can polish on its own.

5. The starting capacitor should be changed with a carbon brush for higher efficiency or machine capability.

\section{References}

1. How to polish with a coconut husk. [cited 2015]. http:// www.ehow.com/how_8708707_polish-coconut-husk.html.

2. Uses of coconut husk. [cited 2014 Aug 29]. http://www. usesofcoconut.com/uses-of-coconut-husk/.

3. Coconut husk floor scrub. [cited 2013 Jan 27]. http:// www.thepackratwifey.com/scrubbing-the-floor-withcoconut-husk/.

4. Industrialfloorcaremachines. [cited2018].https://in.linkedin. com/company/industrial-floorcare-machines-uk. 